\title{
Chapter 42 \\ Genomic Approaches Towards Durable Fungal Disease Resistance in Wheat
}

\author{
Beat Keller, Simon Krattinger, Liselotte Selter, Chauhan Harsh, Jyoti Singla, \\ Susanne Brunner, Severine Hurni, Daniel Stirnweis, Margarita Shatalina, \\ Thomas Wicker, Francis Parlange, Joanna Risk, and Evans Lagudah
}

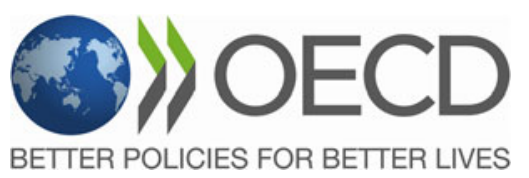

The opinions expressed and arguments employed in this publication are the sole responsibility of the authors and do not necessarily reflect those of the OECD or of the governments of its Member countries.

The Special Session was sponsored by the OECD Co-operative Research Programme on Biological Resource Management for Sustainable Agricultural Systems, whose financial support made it possible for most of the invited speakers to participate in the Special Session.

\begin{abstract}
In the last years there has been enormous progress in the molecular understanding of fungal disease resistance in plants. Research on effector-based immunity which is mediated by major resistance $(R)$ genes has been greatly stimulated by the molecular isolation of plant resistance genes as well as the first fungal effectors. In addition, the first genes underlying QTLs or partial disease resistance have been cloned. However, much of this work is still in a phase of basic research and there is a need for translational approaches to realize the globally needed improvements of disease resistance in wheat. In particular, it is essential that future strategies are aiming at achieving durable resistance against pathogens. Durable resistance has been defined by Johnson (Genetic background of durable resistance.
\end{abstract}

B. Keller $(\bowtie) \bullet$ S. Krattinger $\bullet$ L. Selter $\bullet$ C. Harsh • J. Singla • S. Brunner

S. Hurni • D. Stirnweis • M. Shatalina • T. Wicker • F. Parlange

Institute of Plant Biology, University of Zurich, Zollikerstrasse 107, 8008 Zurich, Switzerland

e-mail: bkeller@botinst.uzh.ch

J. Risk • E. Lagudah

CSIRO Plant Industry, GPO Box 1600, Canberra, ACT 2601, Australia 
In: Lamberti F, Waller JM, Van der Graaff NA (eds) Durable resistance in crops. Plenum, New York, pp 5-24, 1983) as a resistance which remains effective in cultivars that are widely grown for long periods and in environments favorable to the disease. In this article we will discuss different molecular strategies towards achieving durable disease resistance in wheat. In particular, our group focuses on the Pm3 allelic series of race-specific powdery mildew $R$ genes and the $L r 34 / Y r 18 / P m 38 /$ $S r 57$ race non-specific multi-pathogen resistance gene.

Keywords Allele mining $\bullet$ Allele pyramiding $\bullet$ Durable disease resistance $\bullet$ Fungal pathogens $\bullet$ Multiline approach $\bullet$ Pathogen genomics

\section{Genomics in Wheat: New Tools and Resources}

New genomic tools have allowed scientists to develop approaches and strategies which are revolutionizing the way specific questions can be tackled in wheat. The availability of partial or complete genomic sequences, although mostly in a nonordered form, allows for a more efficient characterization of agronomically important genes and to explore their use in classical or transgenic wheat breeding. Map-based isolation of genes relevant for agronomic traits and the study of their allelic diversity have become simpler and faster. In particular, the critical problem of insufficient marker coverage in a targeted region can now be approached more efficiently. In a recent study, we have shown that chromosome sorting performed in the group of J. Dolezel and H. Simkova at the Institute of Experimental Botany in Olomouc, combined with next generation sequencing can be successfully used to efficiently increase the number of single nucleotide polymorphism (SNP) markers in a specific chromosomal region (Shatalina et al. 2013). In this case, the targeted resistance QTL against Stagonospora glume blotch is located on chromosome 3B, the only chromosome that can be isolated in pure form because of its size. However, we have recently found that the same approach can also be used in fractions which are not pure but enriched for a specific chromosome, even if only $25 \%$ of the sample consists of the target chromosome (Singla et al., unpublished data). Although we are only at the beginning of exploiting these new tools, there are already a number of applications which are of direct use and that increase the efficiency of resistance improvement in wheat.

\section{Natural Diversity as a Rich Resource for Basic and Applied Wheat Genetics}

The study of natural diversity at the molecular level in the form of molecular allele mining does not only allow for the identification of new functional disease resistance alleles, but also for the characterization of the molecular basis of resistance 
gene function and specificity. By screening about 2,000 landraces from different geographical regions we were able to increase the number of known functional Pm3 alleles from seven to 16 (Bhullar et al. 2009, 2010).

Based on the available large dataset of functional and non-functional Pm3 alleles, we could derive a hypothesis on functionally important amino acids in this protein. In particular, we focused on the nucleotide-binding-site domain (NBS) of the PM3 protein. Alleles with a broad and a narrow resistance spectrum have been described. We found that a broad Pm3-spectrum range correlates with a fast and intense hypersensitive response (HR) in a Nicotiana benthamiana transient-expression system. This activity can be attributed to two particular amino acids in the ARC2 subdomain of the NBS. The combined substitution of these two amino acids in narrow-spectrum Pm3 alleles enhances their capacity to induce a HR in Nicotiana and, very interestingly, we found that the same substitutions also broaden the resistance spectrum of the $P m 3 f$ allele in wheat, resulting in an improved version of this gene. These results demonstrate the possibility for improvement of the NBS-"molecular switch" acting in the conversion of initial pathogen perception by the LRR into resistance-protein activation. Thus, we have found a way to enhance the resistance spectrum of an existing gene via minimal targeted modifications in the NBS domain. Ultimately, this might also allow the design of synthetic genes with new specificities and, ideally, to develop a more durable type of resistance based on major genes. The application of such findings could be made with transgenic lines, but given that only very small changes are needed in the protein sequence, new technologies such as transcription activator-like effector nucleases (TALEN) and related approaches might be used for gene editing in wheat.

Natural diversity also reveals mechanism of resistance gene evolution. We have found that the $\operatorname{Pm} 8$ gene derived from rye and present on the frequently used 1RS/1BL translocation in wheat is an ortholog of the wheat $P m 3$ resistance gene (Hurni et al. 2013). The finding that orthologous genes have maintained their function against the mildew pathogen over an estimated seven million years revealed a surprising evolutionary stability of powdery mildew resistance gene activity. This is even more surprising given the fact that after the introduction of $P m 8$ in cultivated wheat lines around 70 years ago, this resistance was rapidly overcome by the pathogen. Thus, we propose that the evolutionary events might have been quite different in the natural grassland ecosystems before agriculture compared to the modern agricultural environments.

\section{Transgenic Strategies for a More Durable Use of Major Resistance Genes}

We have recently explored the transgenic use of the $P m 3$ resistance alleles in wheat resistance breeding. We have isolated the $P m 3 a-g$ alleles from different wheat lines and transformed them into the wheat genotype Bobwhite S26 under control of the maize ubiquitin promoter. A large field trial performed in Switzerland in the years 
2008-2010 has shown that most of these alleles confer improved resistance to powdery mildew when overexpressed (Brunner et al. 2011). In particular, it is noteworthy that some transgenic lines had an improved resistance compared to the donor line with the same gene, indicating that overexpression can improve resistance activity.

Moreover, resistance was improved when transgenic lines with different functional Pm3 alleles were mixed in the field (Brunner et al. 2012). This so-called multiline approach has been classically used in agro-ecosystems. However, the lines were never completely isogenic as the different resistance genes were introduced into the same genotypes by backcross breeding, resulting in relatively large chromosomal segments from the donors. Using a transgenic approach, we established true isogenic lines in the same genotype for a number of different Pm3 alleles, with different race spectrum of resistance to wheat powdery mildew. We found a clear improvement of resistance in mixed stands containing lines carrying two different Pm3 alleles, demonstrating the effectiveness of this approach.

The availability of many Pm3 alleles in transgenic form also allowed us to pyramid two alleles in the same genotype. Whereas in classical breeding alleles can only be combined temporarily in $F_{1}$ hybrids, the transgenes have inserted at different locations in the genome and can therefore be combined in a stable, homozygous form after crosses and selection for the presence of two or more alleles in the same plant. We are currently studying the different double homozygous lines for several allelic combinations. We find situations of the expected additivity of gene function, but also interference. The molecular analysis of these lines is ongoing and promises to give fundamental new insight into resistance gene function.

Although the three approaches (overexpression, multilines, gene pyramidization) described above for the transgenic use of major $R$ genes are still in an early phase in relation to applied wheat breeding, they show the potential of such new strategies. In particular it should be considered that wild relatives of crop plants hold great gene/allelic diversity for resistance. It is often difficult and very timeconsuming to transfer genes from wild species into crops by classical breeding: it can take decades to derive agronomically useful material from such introgressions. Transgenic technology, together with a more rapid identification of the relevant genes in the wild germplasm, provide a promising way to use the natural diversity more efficiently and to rapidly develop pre-breeding material for further use.

\section{Molecular Studies on the Durable Wheat Resistance Gene Lr34/Yr18/Pm38/Sr57}

Lr34/Yr18/Pm38/Sr57 (subsequently referred to as Lr34) is a single wheat gene that confers durable and partial adult plant resistance against the four biotrophic diseases leaf rust, stripe rust, powdery mildew and stem rust. This phenotype is also referred to as slow-rusting or slow-mildewing and is linked to leaf tip necrosis, a 
morphological marker associated with senescence-like processes. Lr34 has been extensively used in wheat breeding for more than a century and no pathogen adaptation has been observed so far. Only a few genes with a similar phenotype have been identified in wheat, namely Lr46/Yr29/Pm39 and Lr67/Yr49/Pm46/Sr55. Lr34 encodes for an ATP-binding cassette (ABC) transporter protein (Krattinger et al. 2009). Members of this conserved protein family transport various substrates across biological membranes. The resistant Lr34 allele, which differs by only two amino acid polymorphisms from the susceptible Lr34 version, evolved after the domestication of hexaploid bread wheat 8,000 years ago through the acquisition of two gain-of-function mutations (Krattinger et al. 2013). An Lr34 ortholog is absent in the closely related cereal barley. We therefore stably transformed the Lr34 gene under its native promoter into barley cultivar Golden Promise (Risk et al. 2013). Interestingly, the gene conferred resistance against barley leaf rust (Puccinia hordei) and barley powdery mildew (Blumeria graminis f.sp. hordei), pathogens that are specific to barley and that do not infect wheat. In contrast to wheat, where Lr34 confers resistance only in adult plants, resistance in barley was already observed at the seedling stage. We also observed a strong leaf tip necrosis phenotype that was already visible in seedlings and that had a negative impact on plant vigor and seed setting. These results demonstrate that Lr34-resistance is transferrable to other cereals. However, tight control of $\operatorname{Lr} 34$ expression is necessary to avoid negative impact on yield. The use of different, tissue- or age-specific promoters might allow generating barley plants with adequate levels of resistance and no impact on yield.

\section{New Tools for Resistance Breeding Based on Pathogen Genomics}

It is known that resistance which is based on $R$ genes or quantitatively acting genes can result in a yield penalty because of the associated costs of resistance. Therefore, new approaches based on completely different resistance mechanisms should also be considered and explored actively. One new strategy might be host-induced gene silencing (HIGS) which is based on RNAi and relies on a process where the presence of an RNAi construct in wheat would result in specific gene silencing in the pathogen. The success of this strategy has already been shown in transient assays (Nowara et al. 2010; Pliego et al. 2013). This approach is not based on endogenous resistance genes in the wheat host, but on sequence information from potentially relevant genes of the pathogen. Essential genes in the pathogen might be targeted by RNAi constructs expressed in wheat, resulting in down-regulation and ideally quantitative resistance against the specific pathogen species. In our group, we have recently developed the necessary genomic tools for such approaches in the wheat powdery mildew pathogen. The complete genomic sequence of the wheat powdery mildew genome is now available and can be used for such pathogen-based resistance strategies (Wicker et al. 2013). 


\section{Conclusions}

The approaches described in this contribution are both based on classical as well as transgenic tools to improve disease resistance breeding in wheat. The transgenic approaches will critically depend on public acceptance and a predictable, efficient regulatory framework. Given the enormous challenges for wheat production in the next decades, it is essential that the wheat scientists globally promote and explain to the public the use of classical and novel tools in breeding.

Acknowledgments This work was supported by grants from the Swiss National Science Foundation (310030B_144081/1), an Advanced Grant of the European Research Council (ERC2009-AdG 249996, Durable resistance) and a grant from the Grains Research and Development Corporation \#CSP000063.

Open Access This chapter is distributed under the terms of the Creative Commons Attribution Noncommercial License, which permits any noncommercial use, distribution, and reproduction in any medium, provided the original author(s) and source are credited.

\section{References}

Bhullar NK, Street K, Mackay M et al (2009) Unlocking wheat genetic resources for the molecular identification of previously undescribed functional alleles at the Pm3 resistance locus. Proc Natl Acad Sci U S A 106:9519-9524

Bhullar NK, Zhang Z, Wicker T et al (2010) Wheat gene bank accessions as a source of new alleles of the powdery mildew resistance gene Pm3: a large scale allele mining project. BMC Plant Biol 10:88

Brunner S, Hurni S, Herren G et al (2011) Transgenic Pm3b wheat lines show resistance to powdery mildew in the field. Plant Biotechnol J 9:897-910

Brunner S, Stirnweis D, Diaz Quijano C et al (2012) Transgenic Pm3 multilines of wheat show increased powdery mildew resistance in the field. Plant Biotechnol J 10:398-409

Hurni S, Brunner S, Buchmann G et al (2013) Rye Pm8 and wheat Pm3 are orthologous genes and show evolutionary conservation of resistance function against powdery mildew. Plant $\mathrm{J}$ 76:957-969

Johnson R (1983) Genetic background of durable resistance. In: Lamberti F, Waller JM, Van der Graaff NA (eds) Durable resistance in crops. Plenum, New York, pp 5-24

Krattinger SG, Lagudah ES, Spielmeyer W et al (2009) A putative ABC transporter confers durable resistance to multiple fungal pathogens in wheat. Science 323:1360-1363

Krattinger SG, Jordan D, Mace E et al (2013) Recent emergence of the wheat Lr34 multi-pathogen resistance: insights from haplotype analysis in wheat, rice, sorghum and Aegilops tauschii. Theor Appl Genet 126:663-672

Nowara D, Gay A, Lacomme C et al (2010) HIGS: host-induced gene silencing in the obligate biotrophic fungal pathogen Blumeria graminis. Plant Cell 22:3130-3141

Pliego C, Nowara D, Bonciani G et al (2013) Host-induced gene silencing in barley powdery mildew reveals a class of ribonuclease-like effectors. Mol Plant Microbe Interact 26:633-642

Risk J, Selter LL, Harsh C et al (2013) The wheat Lr34 gene provides resistance against multiple fungal pathogens in barley. Plant Biotechnol J 11:847-854 
Shatalina M, Wicker T, Buchmann JP et al (2013) Genotype-specific SNP map based on whole chromosome 3B sequence information from wheat cultivars Arina and Forno. Plant Biotechnol J 11:23-32

Wicker T, Oberhaensli S, Parlange F et al (2013) The wheat powdery mildew genome shows the unique evolution of an obligate biotroph. Nat Genet 45:1092-1096 